\title{
PROGNOSTIC BIOMARKERS IN EARLY-STAGE B-CLL PATIENTS
}

\author{
Vanya S. Popova, \\ Svetla O. Blajeva1, \\ Margarita L. Alexandrova ${ }^{2}$, \\ Tsvetan H. Lukanov', \\ Silvia Y. Naneva, \\ Nikolay T. Tzvetkov \\ Division of Haematology, \\ Medical University - Pleven, \\ Bulgaria \\ ${ }^{1}$ Department of Clinical Laboratory, \\ Clinical Immunology and \\ Allergology, \\ Medical University - Pleven, \\ Bulgaria \\ ${ }^{2}$ Department of Physics and \\ Biophysics, Preclinical and Clinical \\ Sciences, \\ Medical University - Pleven, \\ Bulgaria
}

\section{Summary}

Chronic lymphocytic leukemia is one of the most common types of leukemia in adults. It belongs to the group of indolent lymphoproliferative disorders and has a slow clinical course. Approximately $50 \%$ of newly diagnosed patients do not require treatment for years. A better understanding of the pathophysiology of the disease has led to the development of models for assessment of the risk.

Our study aimed to evaluate the prognostic significance of the serum marker beta-2 microglobulin (B2M) and the flow cytometric marker CD49d in patients with early-stage of B-chronic lymphocytic leukemia (B-CLL) as well as look for a correlation between CD $49 \mathrm{~d}$ and the early stages of the disease. For this purpose, analysis of 30 untreated patients with known and newly diagnosed B-CLL was carried out. The following methods were used: documentary, flow cytometric analysis of peripheral blood, Rai staging system and chi-square test of independence (Fisher's Exact Test). The results from our study showed that a small number of patients in the early stage of the disease have high levels of CD49d expression and beta- 2 microglobulins. In eight of 29 patients, the flow cytometric marker was higher than $30 \%$, and in ten out of 29 patients, the B2M was above the reference range. No significant correlation between the two markers in early stage B-CLL patients was found.

Key words: microglobulin, leukemia, stage, Rai, risk

\section{Introduction}

Chronic lymphocytic leukemia (CLL) is a disease characterized by a variable clinical course [1]. The patients are classified into two groups: early and advanced stages. Patients are also divided into three groups, using Rai staging system (clinical-topographic) and Binet (laboratory) for evaluating the risk as low, intermediate and high. The aggressive course of the disease is usually associated with advanced clinical stages (III, IV Rai /C-Binet), large tumor mass, high lactate dehydrogenase levels and diffuse bone marrow infiltration. These signs are the so-called "classical" prognostic factors. Over the years, with the introduction of more precise and detailed methods of laboratory evaluation, new biological prognostic markers were 
added. These reflect the process related to cell proliferation, adhesion, apoptosis, and tumor invasion. The serum markers thymidine-kinase, beta-2 microglobulin, soluble CD23, flow cytometric markers CD38, CD49d, ZAP-70 and molecular IgVH mutation status, del 11q, 17p, $13 q$ belong to this group [2].

The $\beta-2$ microglobulin is a low molecular weight protein present in all nucleated cells, and it is a part of the major histocompatibility complex class I molecules $(3,4]$. It was first described in 1968 by Berggard in patients with Wilson's disease [5]. Low levels of beta2 microglobulin in physiological condition can be detected in serum and body fluids of healthy individuals $[3,6,7]$. Typically, the protein is filtered into the glomeruli, reabsorbed and catabolized in the proximal tubules [7,8]. Elevated serum marker values are described in kidney disease associated with glomerular damage, autoimmune and malignant diseases, and viral infections [8]. In cases of malignant hematological diseases, the serum marker is used as a single prognostic factor in patients with malignant lymphoproliferative disorders and is part of the accepted international staging system (ISS) in multiple myeloma patients [9]. Longterm clinical trials confirm its predictive value in follicular lymphoma patients [10]. In medical reviews, B2M correlates with the clinical stages of B-CLL [2].

CD49d belongs to the group of integrins and, together with B-cell receptor (BCR), Toll-like receptors, cytokines and chemokines it supports the cell-cell, cell-matrix interactions and helps the proliferative activity of lymphocytes [11]. It takes part in the formation of macromolecular complexes in the lipid rafts of the B-cell membrane with CD38, CD44, and MMP-9. CD49d is a phenotypic marker, whose high expression is related to poor prognosis [12]. According to medical reviews, the high levels of expression of the marker above $30 \%$ indicate that treatment is to be started [13].

\section{Materials and Methods}

To confirm B-CLL diagnosis, as well as to distinguish CD49d positive patients flow cytometric analysis of peripheral blood of 30 patients was carried out, using FAC Sort/
Becton-Dickinson/ and software product Cell Quest CD 45/ CD 14/ CD 19CD 20/ CD 5 / CD 22/CD 23/ CD 10/HLA- DR/ CD 25/CD 11c/ CD 11a/ CD38/CD 3 / CD 4/ CD 8 / CD 2 /s Ig D / s Ig G / s Ig M/kappa/ lambda. Leukocytes were analyzed by using a dual-laser FACS Calibur cytometer (Becton Dickinson, Heidelberg, Germany) and Cell Quest Pro software (Becton Dickinson). Briefly, blood cells were stained with fluorescence-conjugated antibodies (FITC and PE). After lysis of erythrocytes (Lysis buffer; Becton Dickinson) and two washes, stained PBMC were re-suspended and fixed with CellFIX (BD Biosciences). Ten thousand of lymphocytes were selected in a forward scatter/side scatter (FSC/SSC) lymphocyte gate and saved together with the other leukocyte populations.

Data are presented as a percentage of the lymphocyte gate. The cytometer was calibrated daily with appropriate single-stained samples for setting compensation. The data obtained were analyzed by FACSComp software $@ 2007$ Becton Dickinson. Fluorescence-conjugated antibodies, detected with Becton Dickinson Pharmingen ${ }^{\mathrm{TM}}$ were used to identify cell population CD19Blymphocytes, CD49dPE, CD29 PerCP. The determination of soluble antigen $\beta_{2}$ microglobulin.(Binding Site) concentration was done using the turbidimetric method with SPAplus ${ }^{\circledR}$ The Rai staging system was applied to stage the patients.

Statistical analysis of the data was made with Statistical Package for Social Science versions 19.0 (SPSS.v.19.0). The chi-square test of independence was applied to determine the existence of an association between categorical variables. The phi coefficient was used to measure the strength and direction of the association between nominal variables. A value of $p<0.05$ was considered statistically significant.

\section{Results}

We present the results of patients with diagnosis B-CLL, meeting the revised criteria of IWGCLL from 2008. All patients signed an informed consent, approved by the ethics committee of MU-Pleven. The cohort included early-stage patients (0, I, II Rai). Ten milliliters of blood was used to evaluate immunological marker 
and serum level of B2M. Levels of CD49d over $30 \%$ were accepted as positive in CD19 positive B-lymphocytes. Levels of B2M higher than 2.34 $\mathrm{mg} / \mathrm{l}$ were interpreted as pathologic.

The study included 30 patients - $12(40 \%)$ females and $18(60 \%)$ males. The average age of the patients was 66.7 (41-84). The distribution of patients by age is presented in Figure 1 .

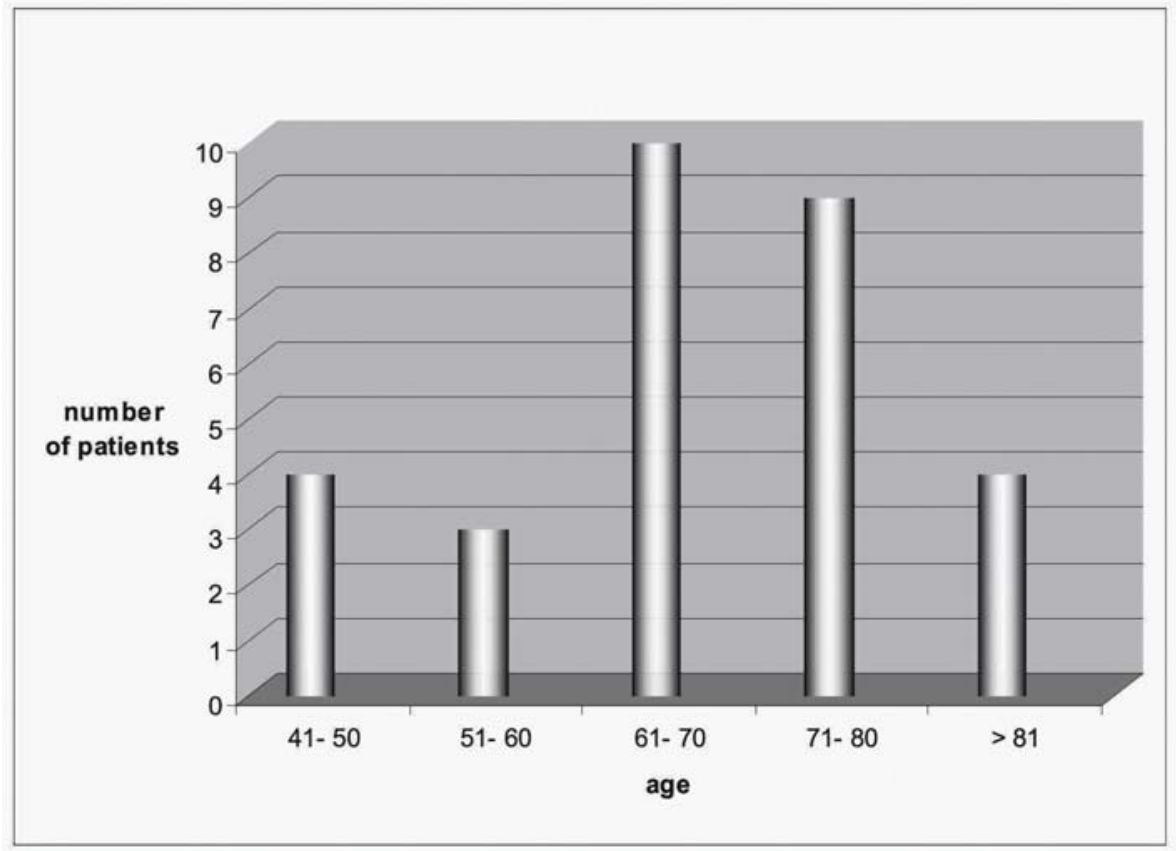

Figure. 1. Age characteristics of the patients

Out of 29 patients (in one of the included patients the immunological marker was not tested), CD49d was positive only in eight of them. Four $(25.0 \%)$ of them were in stage O-Rai, two in stage I, and two $(28.6 \%)$ were in stage II. The distribution of the patients according to stages and CD49d is shown in Table 1.

Table 1. Patient distribution based on stage and CD49d

\begin{tabular}{lll}
\hline \multirow{2}{*}{ Rai stage } & \multicolumn{1}{l}{ CD49d } & \\
\cline { 2 - 3 } & $>\mathbf{3 0 \%}$ & $<\mathbf{3 0 \%}$ \\
\hline 0 stage & $4(25.0 \%)$ & $12(75.0 \%)$ \\
\hline I stage & $2(33.3 \%)$ & $4(66.7 \%)$ \\
\hline II stage & $2(28.0 \%)$ & $5(71.4 \%)$ \\
\hline
\end{tabular}

The test of independence (cross tabulation) was applied to determine whether there was an association between the stage of the disease and CD49dwas applied. The analysis showed no significant difference between CD49d and the early stages of the disease (Fisher's Exact Test $=0.429, \mathrm{p}>0.05$ ).

In one of all the 30 patients, B2M was not tested. To avoid compromising the results, since most of the patients were over 50 years of age; all the patients included in the study were evaluated for additional creatinine clearance. Ten of the early stage patients had serum levels of B2M above the normal range. In 19 individuals, the result was within the standard limits. No significant correlation between $\mathrm{Cd} 49 \mathrm{~d}$ and $\mathrm{B} 2 \mathrm{M}$ was found in the early stage B-CLL patients (Phi coefficient $\varnothing=0.059, \mathrm{p} \leq 0.05$ ).

\section{Discussion}

The results of our study showed that a small number of patients in the early stages of the disease had an elevated beta- 2 microglobulin level, and even fewer had high levels of CD49d in combination with beta-2 microglobulin. The results we obtained are consistent with data reported by M. Gentile et al. (2009). In a group of 222 patients in the early stages of the 
disease (Binet A), only 73 (32\%) had serum marker levels above the reference range. In the same cohort, which was followed for 3.5 years, just in 55 patients $(32 \%)$, it was necessary to start treatment [14]. In our study, out of the 29 patients tested for a serological marker, the B2M was higher than the reference range only in 10 of them. Sarris et al. (2013) investigated the clinical significance of serum levels of free serum light chains in patients with B-CLL. They also reported the existence of a statistically significant relationship between beta- 2 microglobulin level and commencement of treatment [15].

We studied a correlation between the stage of the disease and the levels of CD49d expression as a factor related to the migration of lymphocytes from the blood circulation and their retention in the secondary lymphatic structures. Only eight out of a total of 29 early-stage patients had levels of the flow cytometric marker above $30 \%$. This finding supports the data published by Baumann et al. (2016), whose study showed that high levels of CD49d expression were found only in $30 \%$ of early stage (0, I-II) patients [16].

\section{Conclusions}

The results of our study showed that a small number of patients in the early stages of the disease had high levels of beta-2 microglobulin and CD49d. The extent to which these two indicators are relevant to the time to treatment is to be studied in the longer-term follow-up in the affected group of patients, as well as in patients who at an advanced stage and have not been treated. The absence of statistically significant correlation between B2M and CD49d could be due to the small number of cases we studied.

\section{Acknowledgements}

This study was a part of a project No 11/2016 funded by Medical University - Pleven.

\section{References}

1. Martinelli S, Cuneo A, Formigaro L, Cavallari M, Lista E, Quaglia MF, et al. Identifying high-risk chronic lymphocytic leukemia: a pathogenesisoriented appraisal of prognostic and predictive factors in patients treated with chemotherapy with or without immunotherapy. Mediterr J Hematol Infect Dis. 2016;8(1):e2016047.

2. Montserat E. New prognostic markers in CLL. Hematology Am Soc Hematol Educ Program, 2006;279-84.

3. Bernier GM. beta 2-Microglobulin: structure, function and significance. Vox Sang. 1980;38(6):323-7.

4. Berrebi A, Shvidel L, Arditti D F, Bassous L, Haran M, Shtalrid M. The Significance of elevated beta 2-Microglobulin (b2-m) in B-CLL: evidence of in vitro b2-m secretion following activation of B-CLL cells. Blood. 2009; 114:4380.

5. Argyropoulos CP, Chen SS, Ng Y-H, Roumelioti ME, Kamran S, Singh PP, et al. Rediscovering beta-2 Microglobulin as a biomarker across the spectrum of kidney diseases. Front Med (Lausanne). 2017;4:73.

6. Bethea M, Forman D. Beta -microglobulin: its significance and clinical usefulness. Ann Clin Lab Sci. 1990;20(3):163-8.

7. Andreucci M, Faga T, Riccio E, Sabbatini M, Pisani A, Michael A. The potential use of biomarkers in predicting contrast-induced acute kidney injury.Int J Nephrol Renovasc Dis. 2016; 9:205-21.

8. Zeng X, Hossain D, Bostwick DG, Herrera GA, Ballester B, Zhang PL. Urinary $\beta 2-$ microglobulin is a sensitive indicator for renal tubular injury. Scholarena Journal (SAJ) of Case Reports. 2014;1(1):1-6:

9. Jimenez-Zepeda VH, Duggan P, Neri P, RashidKolvearF, Tay J, Bahlis NJ. Revised International Staging System applied to real world multiple myeloma patients. Clin Lymphoma Myeloma Leuk. 2016;16(9):511-8.

10. Press OW, Unger JM, Rimsza LM, Friedberg JW, LeBlanc M, Czuczman MS, et al. A comparative analysis of prognostic factor models for follicular lymphoma based on a phase III trial of CHOP-Rituximab vs CHOP + ${ }^{131}$ Iodine-Tositumomab. Clin Cancer Res. 2013 Dec; 19(23).

11. Dal Bo M, Tissino E, Benedetti D, Caldana C, Bomben R, Poeta GD, et al. Functional and clinical significance of the Integrin Alpha Chain CD49d expression in chronic lymphocytic leukemia. Curr Cancer Drug Targets. 2016;16(8):659-68.

12. Zucchetto A, Vaisitti $\mathrm{T}$, Benedetti $\mathrm{D}$, Tissino E, Bertagnolo V, Rossi D, et al. The CD49d/ CD29 complex is physically and functionally associated with $\mathrm{CD} 38$ in B-cell chronic lymphocytic leukemia cells. Leukemia. 2012;26(6):1301-12. 
13. Bulian P, Shanafelt TD, Fegan C, Zucchetto A, Cro L, Nückel H, et al. CD49d is the strongest flow cytometry-based predictor of overall survival in chronic lymphocytic leukemia. J. Clin. Oncol. 2014;32(9):897-904.

14. Gentile M, Cutrona G, Neri A, Molica S, Ferrarini M, Morabito F. Predictive value of $\beta 2$-microglobulin $(\beta 2-\mathrm{m})$ levels in chronic lymphocytic leukemia since Binet A stages. Haematolica. 2009; 94(6):887-8.
15. Sarris K, Maltezas D, Koulieris E, Bartzis V, Tzenou T, Sachanas S, et al. Prognostic significance of serum free light chains in chronic lymphocytic leukemia. Clin Adv Hematol. Oncol. 2013 Oct 29;ID 359071.

16. Baumann T, Delgado J, Santacruz R, MartínezTrillos A, Rozman M, Aymerich M, et al. CD49d (ITGA4) expression is a predictor of time to first treatment in patients with chronic lymphocytic leukemia and mutated IGHV status. $\mathrm{Br} \mathrm{J}$ Haematol. 2016;172(1):48-55. 